\title{
Chromosomal Q-Heterochromatin Regions in Individuals of Various Age Groups
}

\author{
A.I. Ibraimov and G.O. Karagulova \\ Laboratory of Human Genetics, National Center of Cardiology and Internal Medicine, Togolok \\ Moldo str., 3, Bishkek, 720040, Kyrghyzstan
}

KEYWORDS Chromosomal Q-heterochromatin; human populations; human adaptation; age groups

ABSTRACT The quantitative content of chromosomal Q-heterochromatin regions was studied in individuals of different age groups taking into account their racial and ethnic affiliation. It was shown that chromosomal Q-HRs are most numerous in the genome of neonates, while they are the least numerous in the genome of elderly subjects (aged 60 years and older) regardless of the ethnic features of the individuals studied. It is supposed that the lesser amount of Q-HRs in the genome of elderly subjects is due to the selective advantage in their survival to old age. The possible selective value of chromosomal Q-HRs is discussed.

\section{INTRODUCTION}

A fundamental feature of chromosomes of higher eukaryotes, including man, is the presence of two evolutionary consolidated types of genetic material: euchromatin and heterochromatin. Euchromatin - the conservative portion of the genome - contains transcribed structural genes, while heterochromatin - the variable portion of the genome - predominantly consist of nontranscribed repeated DNA sequences.

Heterochromatin is universally distributed in the chromosomes of all the higher eukaryotes, amounting to $10 \%-60 \%$ of their genome. About $15 \%-20 \%$ of the human genome are composed of heterochromatin regions (HR) (John 1988). Chromosomal HRs do not change during ontogenesis and are clearly inherited as discrete features.

To-date, two types of heterochromatin are known: C- and Q-heterochromatin. There are several significant differences between them, including the fact that $\mathrm{C}$-heterochromatin is encountered in chromosomes of all higher eukaryotes, while Q-heterochromatin is only present in man, the chimpanzee and gorilla. In man C-heterochromatin is present in all his chromosomes, varying mainly in size, while Qheterochromatin can only be detected on seven autosomes and the Y-chromosome (Paris Conference 1971;, 1975).

Individuals in a population differ from each other in the number, location, size and intensity of staining (fluorescence) of chromo-somal Q- heterochromatin regions (Q-HR). It has been shown that certain human populations differ significantly as concerns this feature (Geraedts and Pearson 1974; Müller et al. 1975; Buckton et al. 1976; Lubs et al. 1977; Yamada and Hasegawa 1978; Al-Nassar 1981; Ibraimov and Mirrakhimov 1985; Stanyon et al. 1988; Kalz et al. 2005). The question remains open as to whether there exist differences between individuals belonging to different age groups (Buckton et al. 1976; Nazarenko 1987). This fact is very important for understanding the possible biological role of the broad quantitative variability of human chromosomal Q-HRs. Therefore, we intended to study using conventional methods Kyrghyz populations, as well as Russian ones belonging to different age groups and living permanently in Bishkek, Kyrghyzstan.

\section{MATERIALS AND METHODS}

Sample Characteristics: Using identical methods we studied neonates, as well as schoolboys and schoolgirls, students, drivers and mechanicians from automobile enterprises in Bishkek aged 7 to 60 years and divided them conditionally into 3 age groups: 1) 7 to 19 years old;2) 20 to 39;3) 40 to 59. The group of elderly subjects (aged 60 years and over) consisted of individuals living in old people's homes of Bishkek.

Cytogenetic Methods: Chromosomal preparations were made using short-term cultures of peripheral blood lymphocytes, with the exception of newborn infants where umbilical 
blood was used. The cultures were processed according to slightly modified (Ibraimov 1983 a) conventional methods (Hungerford 1965). The due used was propylquinacrine mustard. All the chromosomal preparations were analyzed by one and the same cytogeneticist (A.I.I.) to investigate chromosomal Q-HR variability. Calculation and registration of chromosomal Q-HRs was performed using the criteria and methods described in detail elsewhere (Ibraimov et al. 1982; Ibraimov et al. 1990).

Quantitative Characteristics of Q-HR Variability and Methods Used for Comparisons: Q-HR variability of autosomes in populations is usually described in the form of four main quantitative characteristics.

1) The distribution of Q-HRs in a population, i.e., distribution of individuals having different numbers of Q-HRs in the karyotype regardless of the location (distribution of Q-HRs), which also reflected the range of Q-HR variability in the population genome.

2) The derivative of this distribution, an important population characteristic, is the mean number of Q-HRs per individual.

3) The frequency of Q-HRs in twelve loci of seven Q-polymorphic autosomes in the population. Despite the fact that in human autosomes there are twelve loci in which Q-HRs can be detected ( 3 cen, 4 cen, 13 p11, 13 p13, 14 p11, 14 p13, 15 p11, 15 p13, 21 p11,21 p13,22 p11, $22 \mathrm{p} \mathrm{13}$ ), individuals with $24 \mathrm{Q}-\mathrm{HRs}$ in their genome could exist, but such cases have not as yet been reported. In individuals of a population the number of Q-HRs on the autosomes usually ranges from zero to ten (Yamada and Hasegawa 1978; Al-Nassar et al. 1981; Ibraimov and Mirrakhimov 1985).

4) The distribution of Q-HRs on autosomes according to their size and intensity of fluorescence (type of Q-variants), estimated as described by Paris Conference $(1971,1975)$.

The $\chi^{2}$ test was used to compare distributions in Table 1-6 and 9-11. The mean numbers of QHR per individual were compared using the Student t-test.

\section{RESULTS}

Table 1, 2, 3, 4, 5 and 6 show the distribution of the numbers and mean number of Q-HRs $(\bar{x})$ on autosomes in five age groups of Kyrghyz and Russian nationality. As can be seen from these tables, in every case neonates are characterized by the highest $\bar{x}$ value, and by a high range of variability in the distribution of Q-HRs as compared to in individuals from older groups and especially from groups of elderly subjects. These differences are statistically significant.

A tendency is noted towards a decrease in the frequency of Q-HRs with age in all the chromosomes, with the exception of autosome 4 containing the lowest amount of Q-HRs in the human population genome (Tables 7 and 8 ). Of

Table 1: Distribution and mean number of Q-HRs per individual in Kyrghyz subjects of different age

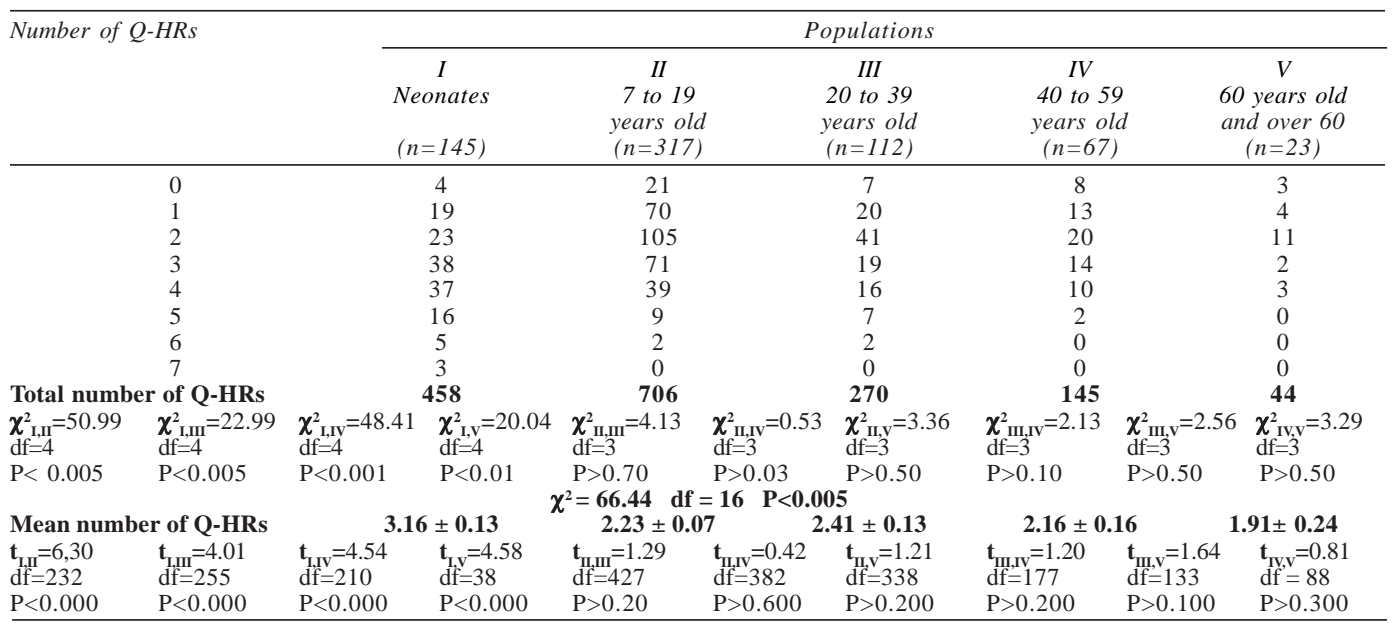


Table 2: Distribution and mean number of Q-HRs per individual in Russian subjects of different age

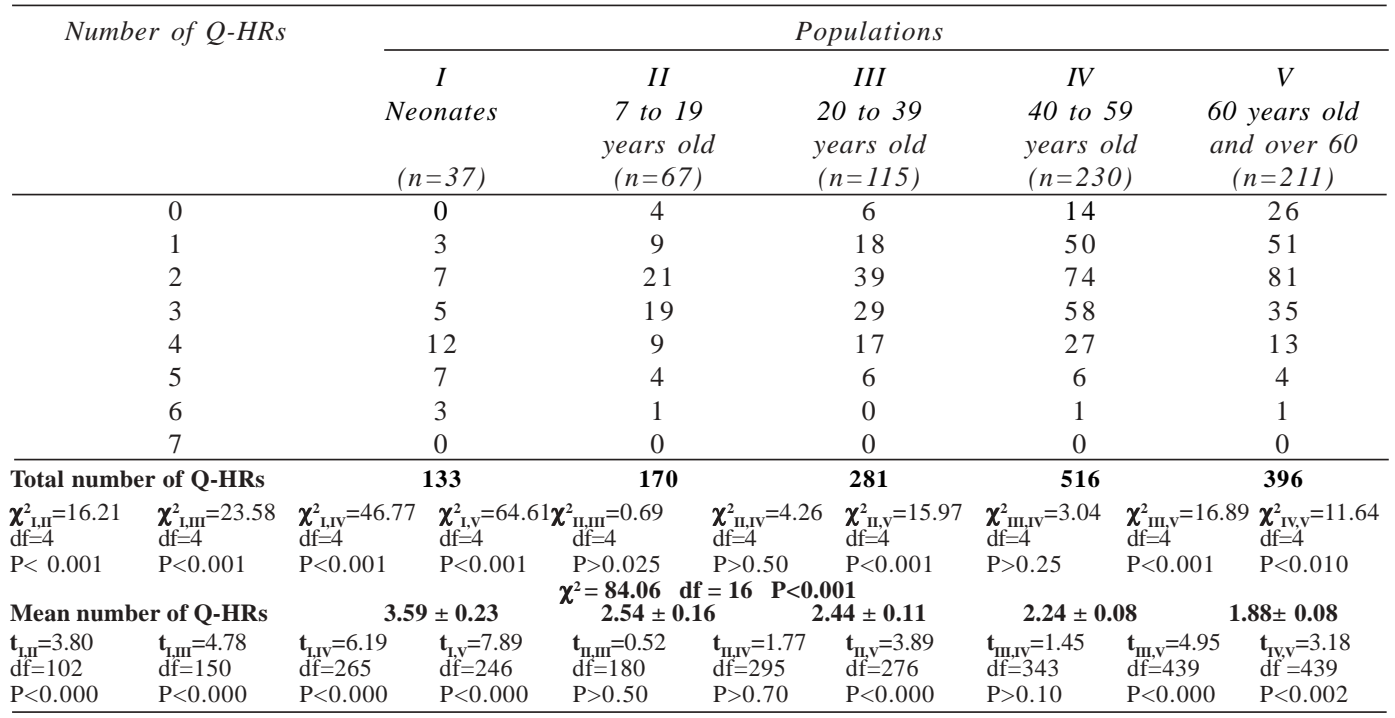

interest is the fact that this difference was pronounced in chromosome 3 and 13 containing more than half the Q-HRs of the human population genome. At the same time, individuals from all the age groups did not differ significantly from each other in the portion of Q-HRs in the polymorphic loci of seven autosomes 3 and 13 (30 and $33 \%$ on average, respectively). The remaining amount of Q-HRs was distributed with various frequencies on autosomes $4,14,15,21$ and 22, in keeping with our previous observations (Ibraimov 1983 b; 1993; Ibraimov et al. 1986; 1990; 1991; 2000; Kalz et al. 2005).

Results of the comparative analyses of the frequency of Q-HRs of various sizes and intensity of fluorescence on autosomes in individuals from different age groups are presented in Table 9 and 10. As can be seen from these Tables, chromosomal Q-HRs are larger and brighter according to the intensity of

Table 3: Distribution and mean number of Q-HRs per individual in Kyrghyz females of different age

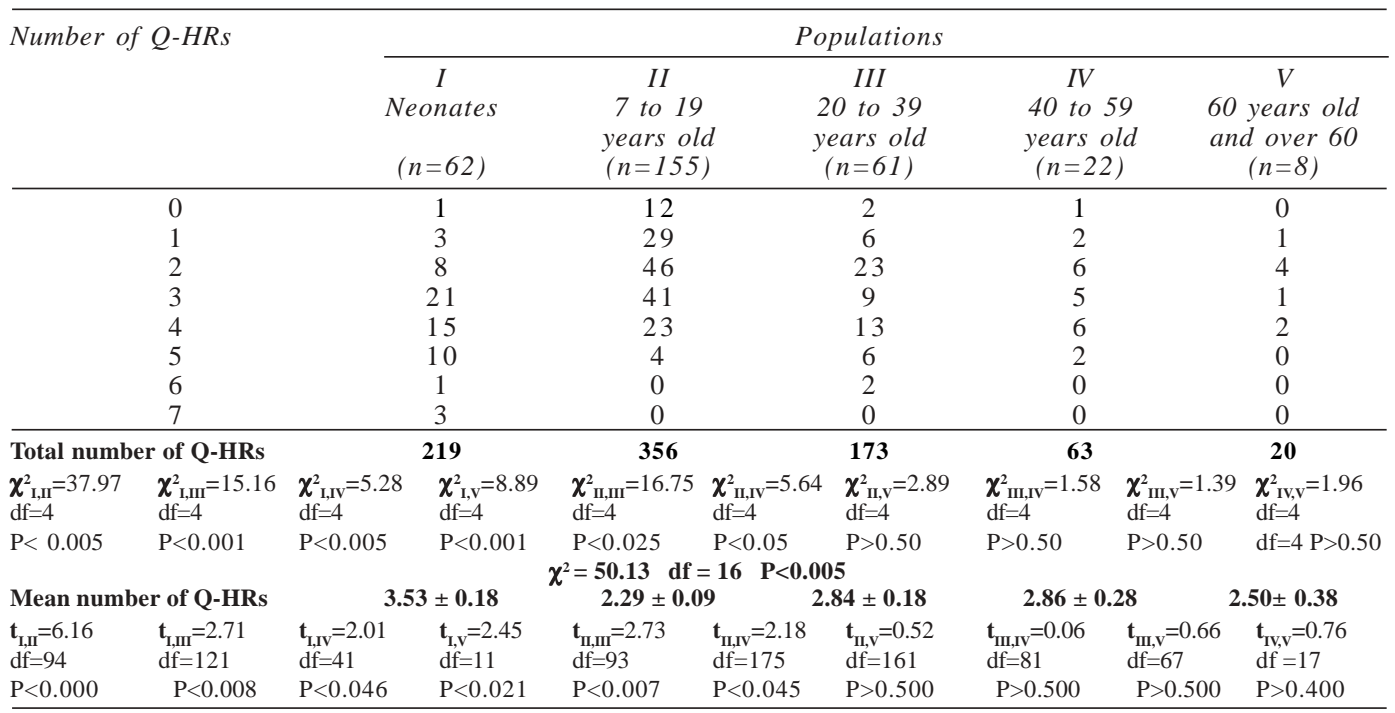


Table 4: Distribution and mean number of Q-HRs per individual in Kyrghyz males of different age

\begin{tabular}{|c|c|c|c|c|c|c|c|c|c|}
\hline \multirow{2}{*}{\multicolumn{2}{|c|}{ Number of $Q-H R s$}} & \multicolumn{8}{|c|}{ Populations } \\
\hline & & \multicolumn{2}{|c|}{$\begin{array}{c}I \\
\text { Neonates } \\
(n=83)\end{array}$} & $\begin{array}{c}\text { II } \\
7 \text { to } 19 \\
\text { years old } \\
(n=162)\end{array}$ & \multicolumn{2}{|c|}{$\begin{array}{c}\text { III } \\
20 \text { to } 39 \\
\text { years old } \\
(n=51)\end{array}$} & \multicolumn{2}{|c|}{$\begin{array}{c}I V \\
40 \text { to } 59 \\
\text { years old } \\
(n=45)\end{array}$} & $\begin{array}{l}\quad V \\
60 \text { years old } \\
\text { and over } 60 \\
(n=15)\end{array}$ \\
\hline \multicolumn{2}{|r|}{0} & \multicolumn{2}{|c|}{3} & 9 & \multicolumn{2}{|r|}{5} & 7 & \multicolumn{2}{|r|}{3} \\
\hline & $\begin{array}{l}1 \\
2 \\
3 \\
4 \\
5 \\
6 \\
7\end{array}$ & & $\begin{array}{c}16 \\
15 \\
17 \\
22 \\
6 \\
4 \\
0\end{array}$ & $\begin{array}{c}41 \\
59 \\
30 \\
16 \\
5 \\
2 \\
0\end{array}$ & & $\begin{array}{l}14 \\
18 \\
10 \\
3 \\
1 \\
0 \\
0\end{array}$ & $\begin{array}{c}11 \\
14 \\
9 \\
4 \\
0 \\
0 \\
0\end{array}$ & & $\begin{array}{l}3 \\
7 \\
1 \\
1 \\
0 \\
0 \\
0\end{array}$ \\
\hline \multicolumn{2}{|c|}{ Total number of Q-HRs } & \multicolumn{2}{|c|}{239} & 350 & & 97 & 82 & \multicolumn{2}{|r|}{24} \\
\hline $\begin{array}{l}\chi_{\mathrm{I,II}}^{2}=28.72 \\
\mathrm{df}=4\end{array}$ & $\begin{array}{l}\chi_{\mathrm{I}, \mathrm{III}}^{2}=17.23 \\
\mathrm{df}=4\end{array}$ & $\begin{array}{l}\chi_{\mathrm{IIVI}}^{2}=15.03 \\
\mathrm{df}=4\end{array}$ & $\begin{array}{l}\chi_{\mathrm{I}, \mathrm{v}}^{2}=11.34 \\
\mathrm{df}=4\end{array}$ & $\begin{array}{l}\chi_{\mathrm{IfIIIII}}^{2}=1.79 \\
\mathrm{df}=4\end{array}$ & $\begin{array}{l}\chi_{\mathrm{IIIIV}}^{2}=3.19 \\
\mathrm{df}=4\end{array}$ & $\begin{array}{l}9 \chi_{\mathrm{II}, \mathrm{V}}^{2}=2.65 \\
\mathrm{df}=4\end{array}$ & 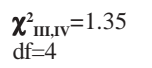 & $\begin{array}{l}\chi_{\mathrm{IIII,V}}^{2}=1.89 \\
\mathrm{df}=4\end{array}$ & $89 \begin{array}{l}\chi_{\mathrm{IN}=\mathrm{V}}^{2}=2.04 \\
\mathrm{df}=4\end{array}$ \\
\hline $\mathrm{P}<0.001$ & $\mathrm{P}<0.001$ & $\mathrm{P}<0.001$ & $\mathrm{P}<0.005$ & $\mathrm{P}>0.05$ & $P>0.05$ & $P>0.05$ & $\mathrm{P}>0.50$ & $P>0.50$ & $P>0.50$ \\
\hline & & & $\chi^{2}=$ & 40.70 df $=16$ & $6 \quad P<0.05$ & & & & \\
\hline Mean num & r of Q-HRs & 2.88 & \pm 0.17 & $2.16 \pm 0.09$ & & $1.90 \pm 0.16$ & $1.82 \pm 0.1$ & & $1.60 \pm 0.29$ \\
\hline $\begin{array}{l}t_{\mathrm{t}, \mathrm{II}}=3.74 \\
\mathrm{df}=130\end{array}$ & $\begin{array}{l}\mathbf{t}_{\mathrm{I}, \mathrm{III}}=4.20 \\
\mathrm{df}=130\end{array}$ & $\begin{array}{l}\mathrm{t}_{\mathrm{I}, \mathrm{IV}}=4.29 \\
\mathrm{df}=113\end{array}$ & $\begin{array}{l}\mathrm{t}_{\mathrm{I}, \mathrm{V}}=3.05 \\
\mathrm{df}=96\end{array}$ & $\begin{array}{l}\mathbf{t}_{\mathrm{nI}, \mathrm{II}}=1.41 \\
\mathrm{df}=211\end{array}$ & $\begin{array}{l}\mathbf{t}_{\mathrm{II}, \mathrm{IV}}=1.74 \\
\mathrm{df}=205\end{array}$ & $\begin{array}{l}\mathbf{t}_{\mathrm{IIIV}}=1.81 \\
\mathrm{df}=175\end{array}$ & $\begin{array}{l}\mathbf{t}_{\mathrm{mIII}, \mathrm{I}}=0.34 \\
\mathrm{df}=94\end{array}$ & $\begin{array}{l}\mathbf{t}_{\mathrm{III}, \mathrm{v}}=0.91 \\
\mathrm{df}=64\end{array}$ & $\begin{array}{c}\mathbf{t}_{\mathrm{rvv},}=0.62 \\
\mathrm{df}=58\end{array}$ \\
\hline $\mathrm{P}<0.000$ & $\mathrm{P}<0.000$ & $\mathrm{P}<0.000$ & $\mathrm{P}<0.003$ & $P>0.100$ & $\mathrm{P}>0.90$ & $\mathrm{P}>0.050$ & $\mathrm{P}>0.700$ & $\mathrm{P}>0.300$ & $\mathrm{P}>0.500$ \\
\hline
\end{tabular}

Table 5: Distribution and mean number of Q-HRs per individual in Russian females of different age

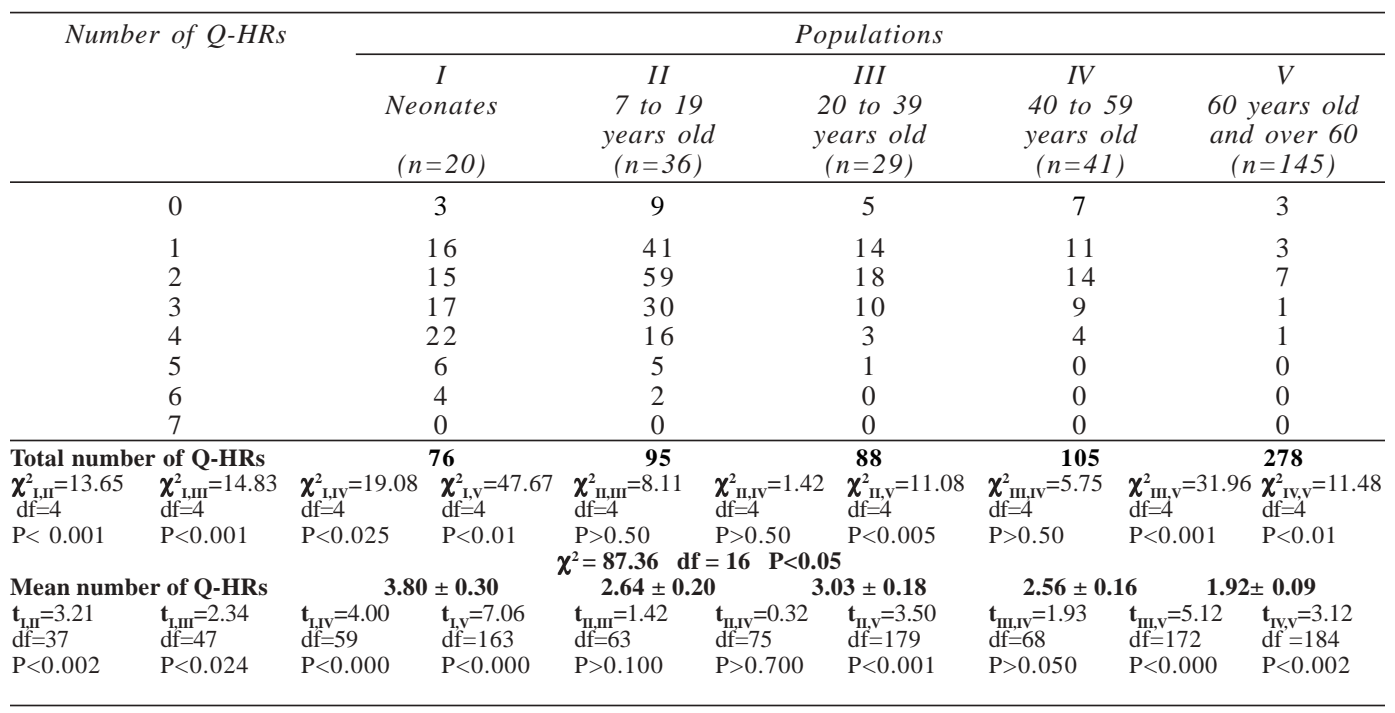

fluorescence in young individuals and especially in neonates.

Tables 11 and 12 show that in younger age groups, on satellite regions ( $\mathrm{p} 13$ ) of acrocentric chromosomes, taken separately and in combination, when Q-HR were simultaneously present on loci p 13 and p 11 of these autosomes, the occurescence of Q-HRs was significantly higher than in elderly subjects, indicating wider quantitative variability of chromosomal Q-HRs in their genome.

\section{DISCUSSION}

Despite the fact that human chromosomal QHRs have been studied for 35 years, their biological role and nature remain unclear (Bhasin 2005).

A remarkable feature of human chromosomal Q-HRs is their wide quantitative variability 
Table 6: Distribution and mean number of Q-HRs per individual in Russian males of different age

\begin{tabular}{|c|c|c|c|c|c|c|c|c|c|}
\hline \multirow{2}{*}{\multicolumn{2}{|c|}{ Number of $Q-H R s$}} & \multicolumn{8}{|c|}{ Populations } \\
\hline & & \multicolumn{2}{|c|}{$\begin{array}{c}I \\
\text { Neonates } \\
(n=17)\end{array}$} & $\begin{array}{c}I I \\
7 \text { to } 19 \\
\text { years old } \\
(n=31)\end{array}$ & \multicolumn{2}{|c|}{$\begin{array}{c}\text { III } \\
20 \text { to } 39 \\
\text { years old } \\
(n=86)\end{array}$} & \multicolumn{2}{|c|}{$\begin{array}{c}I V \\
40 \text { to } 59 \\
\text { years old } \\
(n=189)\end{array}$} & $\begin{array}{c}V \\
60 \text { years old } \\
\text { and over } 60 \\
(n=66)\end{array}$ \\
\hline & 0 & & 0 & 3 & & 6 & 14 & & 9 \\
\hline & 1 & & 2 & 4 & & 16 & 43 & & 17 \\
\hline & 2 & & 3 & 10 & & 33 & 61 & & 24 \\
\hline & 3 & & 4 & 8 & & 19 & 45 & & 13 \\
\hline & 4 & & 5 & 4 & & 6 & 20 & & 2 \\
\hline & 5 & & 1 & 1 & & 6 & 5 & & 0 \\
\hline & 6 & & 2 & 1 & & 0 & 1 & & 1 \\
\hline & 7 & & 0 & 0 & & 0 & 0 & & 0 \\
\hline \multicolumn{2}{|c|}{ Total number of Q-HRs } & & 57 & 75 & & 193 & 411 & & 118 \\
\hline $\begin{array}{l}\chi_{\mathrm{I,II}}^{2}=4.49 \\
\mathrm{df}=4\end{array}$ & $\begin{array}{l}\chi_{\mathrm{I}, \mathrm{III}}^{2}=11.46 \\
\mathrm{df}=4\end{array}$ & $\begin{array}{l}\chi_{\mathrm{I}, \mathrm{IV}}^{2}=14.95 \\
\mathrm{df}=4\end{array}$ & $\begin{array}{l}\chi_{\mathrm{f}, \mathrm{v}}^{2}=23.07 \\
\mathrm{df}=4\end{array}$ & $\begin{array}{l}\chi_{\mathrm{III,III}}^{2}=1.39 \\
\mathrm{df}=4\end{array}$ & $\begin{array}{l}\chi_{\mathrm{IIIIV}}^{2}=1.49 \\
\mathrm{df}=4\end{array}$ & $\begin{array}{l}\chi_{\mathrm{IIIV}}^{2}=7.20 \\
\mathrm{df}=4\end{array}$ & $\begin{array}{l}\chi_{\mathrm{IIIIIV}}^{2}=3.92 \\
\mathrm{df}=4\end{array}$ & $\begin{array}{l}\chi_{\text {fIII, }}^{2}=5.92 \\
d f=4\end{array}$ & $\begin{array}{l}\chi_{\mathrm{IVVV}}^{2}=5.62 \\
\mathrm{df}=4\end{array}$ \\
\hline $\mathrm{P}<0.005$ & $\mathrm{P}<0.001$ & $\mathrm{P}<0.005$ & $\mathrm{P}<0.01$ & $P>0.50$ & $\mathrm{P}>0.25$ & $\mathrm{P}<0.05$ & $P>0.05$ & $\mathrm{P}<0.05$ & $\mathrm{P}<0.05$ \\
\hline \multicolumn{10}{|c|}{$\chi^{2}=28.54 \quad \mathrm{df}=16 \quad \mathrm{P}<0.01$} \\
\hline $\begin{array}{l}\mathbf{t}_{\mathrm{l}, \mathrm{II}}=2.16 \\
\mathrm{df}=46\end{array}$ & $\begin{array}{l}\mathbf{t}_{1, \mathrm{III}}=3.34 \\
\mathrm{df}=101\end{array}$ & $\begin{array}{l}\mathbf{t}_{\mathrm{t}, \mathrm{IV}}=3.70 \\
\mathrm{df}=204\end{array}$ & $\begin{array}{l}\mathbf{t}_{\mathrm{l}, \mathrm{v}}=4.73 \\
\mathrm{df}=81\end{array}$ & $\begin{array}{l}\mathbf{t}_{\mathrm{II}, \mathrm{II}}=0.67 \\
\mathrm{df}=115\end{array}$ & $\begin{array}{l}\mathbf{t}_{\mathrm{IIIV}}=1.04 \\
\mathrm{df}=218\end{array}$ & $\begin{array}{l}.24 \pm 0.15 \\
\mathbf{t}_{\mathrm{II}, \mathrm{v}}=2.33 \\
\mathrm{df}=95\end{array}$ & $\begin{array}{l}\mathbf{t}_{\mathrm{III,IV}}=0.44 \\
\mathrm{df}=273\end{array}$ & $\begin{array}{l}\mathbf{t}_{\mathrm{IIIV}, \mathrm{v}}=2.29 \\
\mathrm{df}=147\end{array}$ & $\begin{array}{c}\mathbf{t}_{\mathrm{Tvv}, \mathrm{v}}=2.21 \\
\mathrm{df}=253\end{array}$ \\
\hline $\mathrm{P}<0.036$ & $\mathrm{P}<0.001$ & $\mathrm{P}<0.000$ & $\mathrm{P}<0.000$ & $P>0.500$ & $\mathrm{P}>0.300$ & $\mathrm{P}<0.022$ & $P>0.600$ & $\mathrm{P}<0.023$ & $\mathrm{P}<0.028$ \\
\hline
\end{tabular}

Table 7: Q-HR frequencies in seven Q-polymorphic autosomes in Kyrghyz subjects of different age

\begin{tabular}{|c|c|c|c|c|c|}
\hline \multirow{2}{*}{$\begin{array}{c}\text { Number of } \\
\text { Q-HRs }\end{array}$} & \multicolumn{5}{|c|}{ Populations } \\
\hline & $\begin{array}{c}I \\
\text { Neonates } \\
(n=145)\end{array}$ & $\begin{array}{c}\text { II } \\
7 \text { to } 19 \\
\text { years old } \\
(n=317)\end{array}$ & $\begin{array}{c}\text { III } \\
20 \text { to } 39 \\
\text { years old } \\
(n=112)\end{array}$ & $\begin{array}{c}I V \\
40 \text { to } 59 \\
\text { years old } \\
(n=67)\end{array}$ & $\begin{array}{c}V \\
60 \text { years old } \\
\text { and over } 60 \\
(n=23)\end{array}$ \\
\hline 3 & $145 \underset{(31.7)^{* *}}{(0.500)^{*}}$ & $\begin{array}{c}251(0.396) \\
(35.6)\end{array}$ & $\begin{array}{c}80(0.357) \\
(29.6)\end{array}$ & $\begin{array}{c}45(0.336) \\
(31)\end{array}$ & $\begin{array}{c}16(0.348) \\
(36.4)\end{array}$ \\
\hline 4 & $\begin{array}{c}16(0.055) \\
(3.5)\end{array}$ & $\begin{array}{c}26(0.041) \\
(3.7)\end{array}$ & $\begin{array}{c}15(0.067) \\
(5.6)\end{array}$ & $\begin{array}{l}6(0.045) \\
(4.1)\end{array}$ & $\begin{array}{l}6(0.130) \\
(13.6)\end{array}$ \\
\hline 13 & $\begin{array}{c}156(0.538) \\
(34.1)\end{array}$ & $\begin{array}{c}206(0.325) \\
(29.2)\end{array}$ & $\begin{array}{c}90(0.402) \\
(33.3)\end{array}$ & $\begin{array}{c}52(0.388) \\
(35.9)\end{array}$ & $\begin{array}{c}18(0.391) \\
(40.9)\end{array}$ \\
\hline 14 & $\begin{array}{c}21(0.072) \\
(4.6)\end{array}$ & $\begin{array}{c}30(0.047) \\
(4.2)\end{array}$ & $\begin{array}{c}15(0.067) \\
(5.6)\end{array}$ & $\begin{array}{c}11(0.082) \\
(7.6)\end{array}$ & $\begin{array}{l}0(0.000) \\
(0.0)\end{array}$ \\
\hline 15 & $\begin{array}{c}55(0.189) \\
(12)\end{array}$ & $\begin{array}{c}72(0.114) \\
(10.2)\end{array}$ & $\begin{array}{c}20(0.089) \\
(7.4)\end{array}$ & $\begin{array}{c}14(0.104) \\
(9.7)\end{array}$ & $\begin{array}{l}0(0.000) \\
(0)\end{array}$ \\
\hline 21 & $\begin{array}{c}36(0.124) \\
(7.9)\end{array}$ & $\begin{array}{c}76(0.119) \\
(10.8)\end{array}$ & $\begin{array}{c}33(0.147) \\
(12.2)\end{array}$ & $\begin{array}{l}8(0.059) \\
(5.5)\end{array}$ & $\begin{array}{l}2(0.043) \\
(4.5)\end{array}$ \\
\hline 22 & $\begin{array}{c}29(0.100) \\
(6.3)\end{array}$ & $\begin{array}{c}45(0.071) \\
(6.4)\end{array}$ & $\begin{array}{c}17(0.076) \\
(6.3)\end{array}$ & $\begin{array}{l}9(0.067) \\
(6.2)\end{array}$ & $\begin{array}{l}2(0.043) \\
(4.5)\end{array}$ \\
\hline $\begin{array}{c}\text { Total number } \\
\text { of Q-HRs }\end{array}$ & 458 & 706 & 270 & 145 & 44 \\
\hline
\end{tabular}

* - Q-HR frequency from the number of chromosomes analysed.

** - Q-HR frequency as percentage of the overall number of chromosomal Q-HR.

characterized by the fact that individuals in a population differ in the number, location, size and intensity of fluorescence of these specific fluorescent areas (Paris Conference 1971, 1975; McKenzie and Lubs 1975). The existence of population Q-HR variability in twelve polymorphic loci of seven autosomes is a wellestablished fact (Geraedts and Pearson 1974;
Müller et al. 1975; Buckton et al. 1976; Lubs et al. 1977; Yamada and Hasegawa 1978; Al-Nassar et al. 1981; Ibraimov and Mirrakhimov 1985; Stanyon et al. 1988; Kalz et al. 2005).

Since it has been first shown by Lubs et al. in 1977 that there exist differences between U.S.A. "whites" and "blacks" in the amount of Q-HRs in their genome, we have studied over 20 human 
Table 8: Q-HR frequencies in seven Q-polymorphic autosomes in Russian subjects of different age

\begin{tabular}{|c|c|c|c|c|c|}
\hline \multirow{2}{*}{$\begin{array}{c}\text { Number of } \\
Q \text {-HRs }\end{array}$} & \multicolumn{5}{|c|}{ Populations } \\
\hline & $\begin{array}{c}I \\
\text { Neonates } \\
(n=37)\end{array}$ & $\begin{array}{c}I I \\
7 \text { to } 19 \\
\text { years old } \\
(n=67)\end{array}$ & $\begin{array}{c}I I I \\
20 \text { to } 39 \\
\text { years old } \\
(n=115)\end{array}$ & $\begin{array}{c}I V \\
40 \text { to } 59 \\
\text { years old } \\
(n=230)\end{array}$ & $\begin{array}{c}V \\
60 \text { years old } \\
\text { and over } 60 \\
(n=211)\end{array}$ \\
\hline 3 & $39(0.527)^{*}$ & $65(0.485)$ & $93(0.404)$ & $186(0.404)$ & $166(0.393)$ \\
\hline 4 & $\begin{array}{l}(29.3)^{* * *} \\
5(0.068) \\
(3.8)\end{array}$ & $\begin{array}{l}(38.2) \\
4(0.029) \\
(2.4)\end{array}$ & $\begin{array}{l}(33.1) \\
3(0.013) \\
(1.1)\end{array}$ & $\begin{array}{l}(36.0) \\
8(0.017) \\
(1.6)\end{array}$ & $\begin{array}{l}(41.9) \\
3(0.007) \\
(0.8)\end{array}$ \\
\hline 13 & $\begin{array}{c}45(0.608) \\
(33.8)\end{array}$ & $\begin{array}{c}57(0.425) \\
(33.5)\end{array}$ & $\begin{array}{c}99(0.430) \\
(35.2)\end{array}$ & $\begin{array}{c}173(0.376) \\
(33.5)\end{array}$ & $\begin{array}{c}127(0.301) \\
(32.1)\end{array}$ \\
\hline 14 & $\begin{array}{l}9(0.122) \\
(6.8)\end{array}$ & $\begin{array}{c}9(0.067) \\
(5.3)\end{array}$ & $\begin{array}{c}20(0.087) \\
(7.1)\end{array}$ & $\begin{array}{c}31(0.067) \\
(6.0)\end{array}$ & $\begin{array}{c}25(0.059) \\
(6.3)\end{array}$ \\
\hline 15 & $\begin{array}{c}10(0.135) \\
(7.5)\end{array}$ & $\begin{array}{c}13(0.097) \\
(7.6)\end{array}$ & $\begin{array}{c}24(0.104) \\
(8.5)\end{array}$ & $\begin{array}{c}38(0.083) \\
(7.4)\end{array}$ & $\begin{array}{l}32(0.076) \\
(8.1)\end{array}$ \\
\hline 21 & $\begin{array}{c}16(0.216) \\
(12.0)\end{array}$ & $\begin{array}{c}15(0.112) \\
(8.8)\end{array}$ & $\begin{array}{c}25(0.109) \\
(8.9)\end{array}$ & $\begin{array}{c}49(0.107) \\
(9.5)\end{array}$ & $\begin{array}{c}23(0.055) \\
(5.8)\end{array}$ \\
\hline 22 & $\begin{array}{l}9(0.122) \\
(6.8)\end{array}$ & $\begin{array}{l}7(0.052) \\
(4.1)\end{array}$ & $\begin{array}{c}17(0.074) \\
(6.0)\end{array}$ & $\begin{array}{l}31(0.067) \\
(6.0)\end{array}$ & $\begin{array}{c}20(0.047) \\
(5.1)\end{array}$ \\
\hline $\begin{array}{l}\text { Total number } \\
\text { of Q-HRs }\end{array}$ & 133 & 170 & 281 & 516 & 396 \\
\hline
\end{tabular}

* - Q-HR frequency from the number of chromosomes analysed.

** - Q-HR frequency as percentage of the overall number of chromosomal Q-HR.

Table 9: Intensity of fluorescence and size of Q-HR in Kyrghyz subjects of different age

\begin{tabular}{|c|c|c|c|c|c|c|c|c|c|}
\hline \multirow{2}{*}{\multicolumn{2}{|c|}{ Number of Q-HRs }} & \multicolumn{8}{|c|}{ Populations } \\
\hline & & \multicolumn{2}{|c|}{$\begin{array}{c}I \\
\text { Neonates } \\
(n=145)\end{array}$} & $\begin{array}{c}\text { II } \\
7 \text { to } 19 \\
\text { years old } \\
(n=317)\end{array}$ & \multicolumn{2}{|c|}{$\begin{array}{c}\text { III } \\
20 \text { to } 39 \\
\text { years old } \\
(n=112)\end{array}$} & $\begin{array}{c}I V \\
40 \text { to } 59 \\
\text { years old } \\
(n=67)\end{array}$ & & $\begin{array}{c}V \\
60 \text { years old } \\
\text { and over } 60 \\
(n=23)\end{array}$ \\
\hline & FQ 14 & & 13 & 661 & & 238 & 137 & & 43 \\
\hline & FQ 15 & & 43 & 45 & & 30 & 8 & & 0 \\
\hline & FQ 25 & & 2 & 0 & & 2 & 0 & & 1 \\
\hline & FQ 35 & & 0 & 0 & & 0 & 0 & & 0 \\
\hline \multicolumn{10}{|c|}{ Total number } \\
\hline \multicolumn{2}{|c|}{ of Q-HRs } & & 58 & 706 & & 270 & 145 & & 44 \\
\hline $\begin{array}{l}\chi_{\mathrm{I}, \mathrm{II}}^{2}=4.64 \\
\mathrm{df}=1\end{array}$ & $\begin{array}{l}\chi_{\mathrm{I}, \mathrm{III}}^{2}=0.74 \\
\mathrm{df}=1\end{array}$ & $\begin{array}{l}\chi_{\mathrm{I}, \mathrm{IV}}^{2}=2.55 \\
\mathrm{df}=1\end{array}$ & $\begin{array}{l}\chi_{\mathrm{I}, \mathrm{V}}^{2}=2.75 \\
\mathrm{df}=1\end{array}$ & $\begin{array}{l}\chi_{\text {IIIIIII }}^{2}=8.06 \\
\mathrm{df}=1\end{array}$ & $\begin{array}{l}\chi_{\mathrm{IIIIV}}^{2}=0.15 \\
\mathrm{df}=1\end{array}$ & $\begin{array}{l}\chi_{\mathrm{IIIV}}^{2}=1.21 \\
\mathrm{df}=1\end{array}$ & $\begin{array}{l}\chi_{\text {IIIIIV }}^{2}=4.35 \\
\mathrm{df}=1\end{array}$ & $\begin{array}{l}\chi_{\mathrm{IIIV}, \mathrm{V}}^{2}=3.69 \\
\mathrm{df}=1\end{array}$ & $\begin{array}{l}\chi_{\mathrm{IV}, \mathrm{V}}^{2}=0.78 \\
\mathrm{df}=1\end{array}$ \\
\hline $\mathrm{P}<0.005$ & $\mathrm{P}<0.050$ & $\mathrm{P}<0.050$ & $\mathrm{P}<0.05$ & $\mathrm{P}<0.05$ & $\mathrm{P}<0.05$ & $\mathrm{P}<0.05$ & $\mathrm{P}>0.50$ & $\mathrm{P}<0.050$ & $\mathrm{P}>0.50$ \\
\hline
\end{tabular}

Table 10: Intensity of fluorescence and size of Q-HR in Russian subjects of different age

\begin{tabular}{|c|c|c|c|c|c|c|c|c|}
\hline \multirow[t]{2}{*}{ Number of $Q-H R s$} & \multicolumn{8}{|c|}{ Populations } \\
\hline & \multicolumn{2}{|c|}{$\begin{array}{c}I \\
\text { Neonates } \\
(n=37)\end{array}$} & $\begin{array}{c}\text { II } \\
7 \text { to } 19 \\
\text { years old } \\
(n=67)\end{array}$ & \multicolumn{2}{|c|}{$\begin{array}{c}I I I \\
20 \text { to } 39 \\
\text { years old } \\
(n=115)\end{array}$} & $\begin{array}{c}I V \\
40 \text { to } 59 \\
\text { years old } \\
(n=230)\end{array}$ & \multicolumn{2}{|c|}{$\begin{array}{c}V \\
60 \text { years old } \\
\text { and over } 60 \\
(n=211)\end{array}$} \\
\hline QFQ 14 & & 11 & 157 & & 259 & 481 & & 368 \\
\hline QFQ 15 & & 22 & 13 & & 22 & 33 & & 28 \\
\hline QFQ 25 & & 0 & 0 & & 0 & 2 & & 0 \\
\hline QFQ 35 & & 0 & 0 & & $0^{`}$ & 0 & & 0 \\
\hline \multirow{3}{*}{$\begin{array}{c}\text { Total number } \\
\text { of } \mathbf{Q}-\mathbf{H R s} \\
\chi_{\mathrm{I}, \mathrm{II}}^{2}=5.78 \\
\mathrm{df}=1 \\
\mathrm{P}<0.05\end{array}$} & & 33 & 170 & & 281 & 516 & & 396 \\
\hline & $\begin{array}{l}\chi_{\mathrm{IfIIII}}^{2}=7.21 \\
\mathrm{df}=1\end{array}$ & $\begin{array}{l}\chi_{\mathrm{IfIV}}^{2}=12.57 \\
\mathrm{df}=1\end{array}$ & $\begin{array}{l}\chi_{\mathrm{I}, \mathrm{V}}^{2}=10.43 \\
\mathrm{df}=1\end{array}$ & $\begin{array}{l}\chi_{\text {IIIIVIV }}^{2}=0.15 \\
\mathrm{df}=1\end{array}$ & $\begin{array}{l}\chi_{\text {II,V }}^{2}=0.05 \\
\mathrm{df}=1\end{array}$ & $\begin{array}{l}\chi^{2}{ }_{\text {IIIIIV }}=0.29 \\
\mathrm{df}=1\end{array}$ & $\begin{array}{l}\chi_{\text {IIIV, }}^{2}=0.14 \\
\mathrm{df}=1\end{array}$ & $\begin{array}{l}\chi_{\mathrm{IVN}, \mathrm{V}}^{2}=0.03 \\
\mathrm{df}=1\end{array}$ \\
\hline & $\mathrm{P}<0.05$ & $\mathrm{P}<0.05$ & $\mathrm{P}<0.05$ & $\mathrm{P}<0.05$ & $\mathrm{P}<0.05$ & $\mathrm{P}>0.50$ & $\mathrm{P}>0.05$ & $\mathrm{P}>0.50$ \\
\hline
\end{tabular}


Table 11: Frequency of Q-HR localized on satellites (p13) and short arms (p11) of acrocentric chromosomes in Kyrghyz subjects of different age

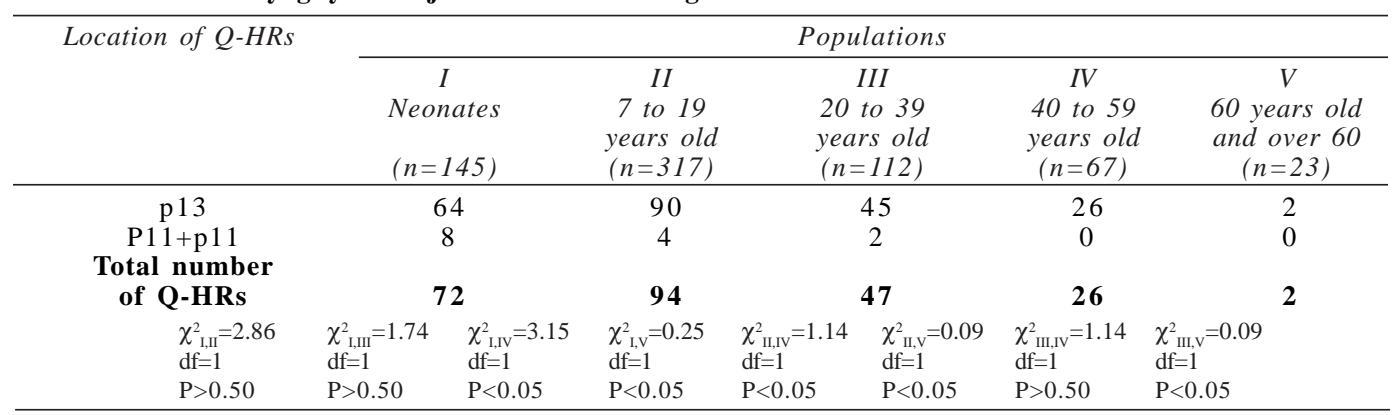

Table 12: Frequency of Q-HR localized on satellites (p13) and short arms (p11) of acrocentric chromosomes in Russian subjects of different age

\begin{tabular}{|c|c|c|c|c|c|c|c|c|}
\hline \multirow[t]{2}{*}{ Type of $Q-H R s$} & & \multicolumn{7}{|c|}{ Populations } \\
\hline & & $\begin{array}{c}I \\
\text { Neonates } \\
(n=37) \\
\end{array}$ & $\begin{array}{c}\text { II } \\
7 \text { to } 19 \\
\text { years old } \\
(n=67)\end{array}$ & \multicolumn{2}{|c|}{$\begin{array}{c}\text { III } \\
20 \text { to } 39 \\
\text { years old } \\
(n=115)\end{array}$} & \multicolumn{2}{|c|}{$\begin{array}{c}I V \\
40 \text { to } 59 \\
\text { years old } \\
(n=230)\end{array}$} & $\begin{array}{c}V \\
60 \text { years old } \\
\text { and over } 60 \\
(n=211)\end{array}$ \\
\hline p 13 & & 24 & 18 & & 53 & 55 & & 38 \\
\hline $\mathrm{p} 11+\mathrm{p} 13$ & & 4 & 3 & & 3 & 4 & & 2 \\
\hline \multicolumn{8}{|l|}{ Total number } & 40 \\
\hline $\begin{array}{l}\chi_{\mathrm{IfII}}^{2}=1.95 \\
\mathrm{df}=1\end{array}$ & $\begin{array}{l}\chi_{\mathrm{I}, \mathrm{III}}^{2}=1.28 \\
\mathrm{df}=1\end{array}$ & $\begin{array}{l}\chi_{\mathrm{IIIV}}^{2}=1.77 \\
\mathrm{df}=1\end{array}$ & $\begin{array}{l}\chi_{\mathrm{I}, \mathrm{V}}^{2}=1.69 \\
\mathrm{df}=1\end{array}$ & $\begin{array}{l}\chi_{\text {IIIIVI }}^{2}=1.09 \\
\mathrm{df}=1\end{array}$ & $\begin{array}{l}\chi_{\text {IIIV, }}^{2}=1.58 \\
\mathrm{df}=1\end{array}$ & $\begin{array}{l}\chi_{\text {IIIIIV }}^{2}=0.10 \\
\mathrm{df}=1\end{array}$ & $\begin{array}{l}\chi_{\text {IIII, }}^{2}=0.01 \\
\mathrm{df}=1\end{array}$ & $\begin{array}{c}\chi_{\mathrm{IVVV}}^{2}=0.13 \\
\mathrm{df}=1\end{array}$ \\
\hline $\mathrm{P}>0.50$ & $\mathrm{P}<0.05$ & $\mathrm{P}<0.05$ & $\mathrm{P}>0.50$ & $\mathrm{P}<0.05$ & $P>0.50$ & $\mathrm{P}>0.50$ & $\mathrm{P}>0.50$ & $\mathrm{P}>0.50$ \\
\hline
\end{tabular}

populations of Eurasia and Africa, representing all the three major racial groups. Within each sample we studied ethnically homogeneous individuals aged 16 to 25 years. These studies showed that certain human populations differ considerably from each other and these differences are mainly related to the environment they live in and not to racial or ethnic features. In particular, the mean number of Q-HRs per individual is considerably lower in the genome of populations living permanently in the North and at high altitude, as well as in newcomers well adapted to extreme environmental conditions of high altitudes (mountaineers) and the Far North (borers - oil industry workers of West Siberia), as compared to populations living in temperate zones of Eurasia and in low-altitude subequatorial Africa (Ibraimov and Mirrakhimov 1982 a, b, c; Ibraimov et al. 1982; 1986; 1990; 1991; 1997; 2000).

Based on these observations, we have at one time put forward the hypothesis on the possible selective value of chromosomal Q-HRs in the adaptation of human populations to certain extreme environmental factors - in particular to cold and hypoxia. These same data are the basis of our cytogenetic model of the origin of contemporary man (Ibraimov 1993). We feel that a conclusive role in this unique process could be played by features of the chromosomal Q-HR system, only inherent to our nearest ancestors and inherited by us, and not by the appearance of new specific structural genes or gene complexes (Ibraimov 1993).

If our assumption on the possible selective value of the amount of chromosomal Q-HRs in human adaptation and the origin of man is correct, than validation of this hypothesis requires, among other things, further reliable data on the amount of Q-HRs in the genome of individuals from different age groups, because we have sufficient reasons to believe that individuals with a lesser amount of Q-HRs in their genome are not only able to adapt themselves better to certain extreme environmental factors, but also have more chances to live to old age.

In the literature we have found only two studies directly or indirectly devoted to chromosomal Q-HR variability in individuals from different age groups. Thus, Buckton et al. (1976) have examined three Scottish populations. Two of the populations, newborn babies and 14 year 
old cohort, were from the Scottish mainland (Edinburgh area). The third population (65 years and over) was from Barra, a relatively isolated island in the Outer Hebrides. The authors came to the conclusion that "rather more variation is found in the Q-band intensity polymorphisms: the island population appears to have fewer Brilliant and intense variants than do the other two groups, 2.9 per person as compared to 4.2 and 3.9 for the newborn and 14 years old subjects, respectively; this may be an age differences rather than a population differences". However, the authors remarked that "these figures may reflect a real phenomenon in that the Barra population shows less polymorphic variation than the mainland populations; but equally well they may reflect a possible age difference, perhaps in the way that chromosomes from aged individuals respond to technical treatment? These possibilities could be evaluated by studying Barra and mainland population samples with a vertical age distribution". Unfortunately, as far as we know, such a study has not yet been carried out in either Scotland or elsewhere.

In Siberia Nazarenko (1987) tried to reveal "age-related changes in the frequency of chromosomal Q-polymorphism in an ethnically homogeneous normal human population". The age of the subjects studied ranged from 7 to 101 years. The author divided them into 3 age groups: 7 to 19 years old, 20 to 29 and 50 years old and over. He found the following changes in the mean number of Q-variants per individual in these 3 age groups: $3.67 \pm 0.10 ; 3.45 \pm 0.14$ and $3.12 \pm$ 0.18 , respectively. Based on these data the author believes that "correlation analysis has shown a low but statistically significant negative linear age-dependence of the mean number of Qvariants per individual".

Two explanations are proposed (Nazarenko 1987) for the somewhat decreased mean number of chromosomal Q-HRs in the older individuals as compared to those under 20 years of age who were similar in other respects: (1) Q-HRs instability during ontogenesis; and (2) the dependence of adaptability and survival upon the location of Q-HRs.

The first hypothesis is to be confirmed by continuous follow-up in a large sample of individuals (from the onset of zygote formation to death). As far as we know, such studies have not been performed. The second hypothesis is not confirmed by our data showed that as a rule, increases in the mean number of Q-HRs are accompanied by increases in absolute Q-HR frequencies on all the autosomes, and vice versa. Populations with a low mean number of O-HRs per individual also have a narrow range of variability in the amount of chromosomal Q-HRs (Ibraimov 1993; Ibraimov et al. 2000). This is also documented by data presented here (Tables 7 and 8).

The published literature devoted to chromosomal Q-HRs suggests that there is as yet no evidence for the existence of basic structural and functional differences between QHRs localized in various chromosomes (Prokofyeva-Belgovskaya 1986; Verma 1988; Bhasin 2005). The results of our former and present analysis favour the suggestion that chromosomal Q-HRs in the genome of human populations behave as a single structural and functional genetic system (Ibraimov 1993; Ibraimov et al. 1986; 2000).

This view is supported by previous works which showed that these specific chromosomal structures, at least in populations living under extreme natural conditions, behave like genetic material subject to natural selection (Ibraimov 1993; Ibraimov and Mirrakhimov 1982 a, b, c; Ibraimov et al. 1982, 1986, 1990, 1991, 1997, 2000). Indeed, if natural selection is to maintain chromosomal Q-HRs as a single structural and functional system, then, (1) in the population there must be individuals with a variable number of QHRs in their karyotype so as to preserve a level of variability, and (2) Q-HR localization on one or another chromosome should not have any significant impact on the viability of the individual (Ibraimov et al. 2000).

The hypothesis on which the project is based assumes that older individuals probably have a lower number of Q-HRs in their genome than younger ones from the same population, since it is known that the amount of Q-HRs does not change during ontogenesis (ProkofyevaBelgovskaya 1986). If this proves to be true, then we have the right to assume that individuals with a smaller amount of Q-HRs in their genome are not only able to adapt themselves better to certain extreme environmental factors, but also have more chances, other things being equal, to live to old age. And indeed, our data indicate that 1 ) in a population there is a clear-cut tendency towards a decrease in the number of chromosomal Q-HRs with age, regardless of racial 
and ethnic features on the individuals (Tables 1,2, $3,4,5$ and 6$) ; 2$ ) of all the age groups the genome of neonates contains the greatest number of QHRs; 3) decreases in the number of Q-HRs with age are not due to the "loss" of Q-heterochromatin on individual loci or chromosomes, but occur simultaneously in all the seven Q-polymorphic autosomes (Tables 7 and 8), in keeping with all our previous observations (Ibraimov 1993; Ibraimov et al. 1986, 1990, 1991, 1997, 2000); 4) decreases QHRs with age concern not only their number, but also their size and intensity of fluorescence (Table 9 and 10); 5) the frequency of Q-HRs localized on satellite regions of acrocentric chromosomes decreases with age (Tables 11 and 12), as it was noted that Q-HRs in these loci occur more frequently in individuals in whom the number of Q-HRs in their genome is greater than on the average in a population (Ibraimov and Mirrakhimov 1985).

Perhaps it should be noted that decreases in the number of chromosomal Q-HRs with age do not occur smoothly apparently. Thus, according to our data, in the Kyrghyz and Russian populations $39.6 \%$ and $47.6 \%$ of chromosomal Q-HRs "disappeared" by the age of 60 years, respectively. Of them the portion of new-born Kyrghyz and Russian infants amounted to $70.6 \%$ and $58.4 \%$, respectively.

In other words, if the number of chromosomal Q-HRs really decreases with age, then this apparently mainly occurs in the early years of life. Therefore we believe that the decrease in the number of Q-HRs with age in a population is not an ontogenetic process, but rather the results of natural selection where by individuals with a greater number of chromosomal Q-HRs in their genome than on the average in a population "fall out".

Therefore, we once again suggest that the cause of the observed differences lies in differential adaptability of individuals with different amounts of Q-heterochromatin material in their genome regardless of its location in any chromosome. We feel that individuals with a high amount of Q-heterochromatin material in their genome are less likely to reach old age.

\section{ACKNOWLEDGEMENT}

The research described in this paper was made possible, in part, by grant number MYR 000 from the International Science Foundation. I am grateful to Dr. S. Tabaldiev for excellent technical assistance, and to an anonymous reviewer for constructive comments.

\section{REFERENCES}

Al-Nassar KE, Palmer CG, Connealy PM, Pao-Lo Yu 1981. The genetic structure of the Kuwaiti population. II. The distribution of Q-band chromosomal heteromorphisms. Hum Genet, 57: 423-427.

Bhasin MK 2005. Human Population Cytogenetics: A Review. Int J Hum Genet, 5(2): 83-152.

Buckton KE, O'Riordan ML, Jacobs PA, Robinson JA, Hill R, Evans HJ 1976. C- and Q-band polymorphisms in the chromosomes of three human populations. Ann Hum Genet, 40: 90-112.

Geraedts JPM, Pearson PL 1974. Fluorescent chromosome polymorphisms: frequencies and segregation in a Dutch population. Clin Genet, 6: 247-257.

Hungerford DA 1965. Leucocytes cultured from small inocula of whole blood and preparation of metaphase chromosomes by treatment with hypotonic $\mathrm{KCl}$. Stain Technol, 40: 333-338.

Ibraimov AI 1983 a. Chromosome preparations of human whole lymphocytes - an improved technique. Clin Genet, 24: 240-242.

Ibraimov AI 1983 b. Human chromosomal polymorphism. VII. The distribution of chromosomal Q-polymorphic bands in different human populations. Hum Genet, 63: 384-391.

Ibraimov AI 1993. The origin of modern humans: a cytogenetic model. Hum Evol, 8: 81-91.

Ibraimov AI, Mirrakhimov MM 1982 a. Human chromosomal polymorphism. III. Chromosomal Qpolymorphism in Mongoloids of northern Asia. Hum Genet, 62: 252-257.

Ibraimov AI, Mirrakhimov MM 1982 b. Human chromosomal polymorphism. IV. Chromosomal Qpolymorphism in Russians living in Kyrghyzia. Hum Genet, 62: 258-260.

Ibraimov AI, Mirrakhimov MM 1982 c. Human chromosomal polymorphism. V. Chromosomal Qpolymorphism in African populations. Hum Genet, 62: 261-265.

Ibraimov AI, Mirrakhimov MM 1985. Q-band polymorphism in the autosomes and the $\mathrm{Y}$ chromosome in human populations. In: AA Sandberg (Ed.): Progress and Topics in Cytogenetics. The $Y$ chromosome. Part A. Basic Characteristics of the $Y$ chromosome. New York: Alan R. Liss Inc., pp 21387.

Ibraimov AI, Mirrakhimov MM, Nazarenko SA, Axenrod EI, Akbanova GA 1982. Human chromosomal polymorphism. I. Chromosomal Q-polymorphism in Mongoloid populations of Central Asia. Hum Genet, 60: 1-7.

Ibraimov AI, Mirrakhimov MM, Axenrod EI, Kurmanova GU 1986. Human chromosomal polymorphism. IX. Further data on the possible selective value of chromosomal Q-heterochromatin material. Hum Genet, 73: 151-156.

Ibraimov AI, Kurmanova GU, Ginsburg EKh, Aksenovich TI, Axenrod EI 1990. Chromosomal Q- 
heterochromatin regions in native highlanders of Pamir and Tien-Shan and in newcomers. Cytobios, 63: $71-82$.

Ibraimov AI, Axenrod EI, Kurmanova GU, Turapov DA 1991. Chromosomal Q-heterochromatin regions in the indigenous population of the northern part of West Siberia and new migrants. Cytobios, 67: 95100 .

Ibraimov AI, Karagulova GO, Kim EY 1997. Chromosomal Q-heterochromatin regions in indigenous populations of the Northern India. Indian J Hum Genet, 3(2): 77-81.

Ibraimov AI, Karagulova GO, Kim EY 2000. The relationship between the $\mathrm{Y}$ chromosome size and the amount of autosomal Q-heterochromatin in human populations. Cytobios, 102: 35-53.

John B 1988. The biology of heterochromatin. In: RS Verma (Ed.): Heterochromatin: Molecular and Structural Aspects. New York: Cambridge University Press, pp.1-147.

Kalz L, Kalz-Fuller B, Hegde S, et al. 2005. Polymorphisms of Q-band Heterochromatin: Qualitative and Quantitative Analyses of Features in 3 Ethnic Groups (Europeans, Indians, and Turks). Int J Hum Genet, 5(2): 153-163.

Lubs HA, Patil SR, Kimberling WJ, Brown J, Hecht F, Gerald P, Summitt RL 1977. Racial differences in the frequency of $\mathrm{Q}$ - and C-chromosomal heteromorphism. Nature, 268: 631-632.

McKenzie WH, Lubs HA 1975. Human Q and C chromosomal variations: distribution and incidence. Cytogenet Cell Genet, 14: 97-115.

Müller HJ, Klinger HP, Glasser M 1975 Chromosome polymorphism in a human newborn population. II. Potentials of polymorphic chromosome variants for characterizing the idiogram of an individual. Cytogenet Cell Genet, 15: 239-255.

Nazarenko SA 1987 Age dynamics of fluorescent polymorphism in human chromosomes. Cytol Genet (Russian), 21: 183-186.

Paris Conference, 1971, and Supplement, 1975. Standartization in Human Cytogenetics . Birth Defects, XI: 1-84.

Prokofyeva-Belgovskaya AA 1986. Heterochromatin Regions of Chromosomes (Russian). , Moscow: Nauka.

Stanyon R, Studer M , Dragone A, De Benedictis G, Brancati C 1988. Population cytogenetics of Albanians in Cosenza (Italy): frequency of Q- and C-band variants. Int J Anthropol, 3: 19-29.

Verma RS (Ed.) 1988. Heterochromatin: Molecular and Structural Aspects. New York: Cambridge University Press.

Yamada K, Hasegawa T 1978. Types and frequencies of Q-variant chromosomes in a Japanese population. Hum Genet, 44: 89-98. 